\section{P-260 DEVELOPING AN ON-LINE TOOLKIT TO SUPPORT END OF LIFE CARE}

${ }^{1}$ Philippa Shreeve, ${ }^{2}$ Ruth England. ${ }^{1}$ Treetops Hospice Care, Derby, UK; ${ }^{2}$ Royal Derby Hospital, Derby, UK

\subsection{6/bmjspcare-2018-hospiceabs.285}

Background When the LCP was removed, a range of materials and guidance required review; furthermore, revised practice needed a platform in order to be shared. We recognised that a new approach might be supported by a novel on-line resource.

Methods A suite of tools that could be used by different providers across Derbyshire at different stages in a patient's end of life journey were collected together. Initially, these documents were held on a webpage hosted by a local community healthcare provider. An accompanying webpage contained details of upcoming educational events and contacts. In the first six months these pages had approximately 6500 hits, with each visit lasting on average $2.5 \mathrm{~min}$. However, feedback stated the pages were not easily accessible to all staff and were clumsy to use. Funding was obtained to build a more 'user friendly' resource. An improved platform was launched in October 2015.

Results In the period 1 October 2016 to 30 September 2017, 9862 sessions were conducted on our website by 5842 users. The average number of pages viewed per session was 3.6 with an average session duration being 3'34'. More than 50\% of users in this period were new to the website and our low bounce rate suggests that people who come to us find what they are looking for. The most popular resource is symptom management guidance. Most activity still comes from desktop computers but users do access the website via mobile devices. It is now possible to create an individualised learning portfolio using the toolkit. This can be linked to existing accredited resources (eELCA) as well as signposting to local face-to-face events.

Conclusions This novel website is a well-used platform for a suite of resources, as well as a means of educational support for those engaging in end of life care.

\section{P-261 ASSESSING THE IMPACT OF INTERVENTIONS TO PROMOTE CLINICAL EFFECTIVENESS IN A PALLIATIVE CARE SERVICE}

${ }^{1}$ Nicola Loveday, ${ }^{2}$ Matthew Curtis. ${ }^{1} T$ The Rowans Hospice, Waterlooville, UK; ${ }^{2}$ The Royal Bournemouth and Christchurch Hospitals NHS Foundation Trust, Bournemouth, UK

\subsection{6/bmjspcare-2018-hospiceabs.286}

Background Clinical effectiveness is a major component of clinical governance and integral to the successful operation of a palliative care service (PCS). Our PCS delivers regular internal educational programmes to share recent developments and latest knowledge and thereby promote high standards of care. Educational theory would suggest that different approaches might suit particular staff groups but it is not clear which approaches are best.

Aims To assess the most effective method to disseminate new learning throughout the PCS. To explore whether professional group is relevant to structure of learning event.

Method Delivery of the same material (highly relevant published paper, pertinent to specialists' practice) using two different approaches.
Session 1: CNS group. Paper distributed to all participants; read before the teaching session. Each allocated a section; presented findings at small group session. Researchers (two doctors) present to facilitate discussion.

Session 2: Doctors of all grades; researchers (same two doctors) presented the paper, reflecting on pertinent points. Structured questions throughout; highly interactive session.

Identical short survey sent before and after intervention to compare clinicians' baseline and post-teaching knowledge; a proxy measure of the effectiveness of said intervention.

Results - Both groups had improved knowledge postintervention.

- Baseline knowledge of CNSs was better than doctors (53\% v. $40 \%$ correct).

- CNSs demonstrated better post-teaching knowledge than doctors (95\% v. 78\%).

Conclusion This work highlights the importance of evaluating the impact of interventions to promote clinical effectiveness and seems to show that one form of intervention may be superior to the other. Future work should now focus on whether this 'more effective' approach is effective for doctors. Due to small numbers of learners, the team plan to continually assess these approaches over a number of topics. Further study to see whether this learning has a positive impact on practice and the outcomes for patients.

\section{P-262 TEN RECOMMENDATIONS FOR ORGANISATIONAL CHANGE TO BETTER SUPPORT CARERS AT END OF LIFE}

${ }^{1}$ Gail Ewing, ${ }^{2}$ Gunn Grande. 'University of Cambridge, Cambridge, UK; ${ }^{2}$ University of Manchester, Manchester, UK

\subsection{6/bmjspcare-2018-hospiceabs.287}

Background Carers play a vital role in supporting patients at end of life (EOL). EOL care policy promotes comprehensive person-centred assessment/support for carers, but without a clear implementation strategy this will only remain an aspiration.

Aim To develop recommendations for organisational structures/ processes required for implementation of comprehensive person-centred assessment/support for carers in EOL practice.

Methods 1) Secondary analysis of qualitative data on implementation in 36 services to identify factors facilitating/hindering implementation. 2) Expert consultation (focus groups with 19 lead practitioners/hospice managers) to review Stage1 findings and develop draft recommendations. 3) Wider stakeholder consultation - two professional workshops (23 participants: hospice, hospital, community, policy and academics), online survey (62 participants) and two carer workshops (nine participants) to finalise recommendations.

Results Comprehensive, person-centred carer assessment/support requires whole-systems change; a cultural shift at practitioner and organisational level. Ten recommendations identify key structures and processes not normally met by current provision:

- Consistent identification of carers within the care setting

- Demographic and contextual data on who the carer is and their situation

- A method/protocol for assessing carers and responding to assessment

- A recording system for carer information (separate from patient data) 